\title{
WLAN/inertial sensors combined positioning system
}

\author{
Zheng Chen ${ }^{1,}$, , TongYu Zhu ${ }^{2, b}$ \\ ${ }^{1}$ State Key Laboratory of Software Development Environment, Beihang University, Beijing, 100191, \\ China \\ ${ }^{2}$ State Key Laboratory of Software Development Environment, Beihang University, Beijing, 100191, \\ China \\ aemail: chenzheng0419@yeah.net, bemail: zhutongyu@nlsde.buaa.edu.cn
}

Keywords: WLAN; inertial sensors; combined positioning system; particle filter

\begin{abstract}
RSS (Received signal strength) floating is the major limit to WLAN (wireless local area network) based positioning accuracy. We proposes an algorithm based on particle filter in order to realize a WLAN/inertial sensors combined positioning system using inertial sensors in the intelligent mobile terminals. The system fuses the complementary information from WLAN and inertial sensors positioning techniques to correct the positioning error due to the RSS floating and the accumulated error of inertial sensors. The experimental results show that the positioning accuracy of the proposed system is reduced by $49 \%$ and $88 \%$ as compared with WLAN based system and inertial sensors based system.
\end{abstract}

\section{Introduction}

With the rapid development of the Internet of Things and smart city construction, LBS (location based services) based application is more and more widely used. Airports, shopping malls, underground parking and so on all require accurate indoor positioning information. At the same time, the mobile terminal is growing rapidly in recent years, and especially intelligent mobile terminals is sought after by more and more users because of its unique advantages.

Positioning applications based on intelligent mobile terminals are mostly applied to relatively open outdoor environment, but it has a poor performance in the indoor environment.

To solve the problem of indoor positioning, the location fingerprinting positioning method based on WLAN attracts a wide range of research. Compared with other indoor positioning system, such as Ultra-Wideband and ZigBee, WLAN based indoor positioning system owns the advantages of low cost, high accuracy and wide range of application. But RSS floating caused by multipath interference seriously deteriorates WLAN positioning accuracy ${ }^{[1,2]}$. Meanwhile, it will take a toll to positioning failure in the area without the coverage of AP (access point), so WLAN positioning needs supplement of other positioning systems.

With inertial sensors are widely used in the intelligent mobile terminal, researches on inertial sensors positioning also become popular. PDR (Pedestrian dead reckoning) is a navigation method using inertial sensors ${ }^{[3]}$. The advantage of PDR is its low cost and high accuracy in short time. And the disadvantage is the presence of accumulated error, so we are unable to obtain long-term stable and reliable positioning information.

We propose an indoor positioning method combining WLAN positioning method and inertial sensors. We use inertial sensors information to correct the errors caused by RSS floating and the lack of Aps. Meanwhile, the WLAN positioning results can help correct accumulated error caused by inertial sensors.

\section{Related Work}

$\mathrm{RADAR}^{[4]}$ protosystem put forward by the wireless network communication group, is the first public positioning system based on WLAN. They used Fingerprinting method to position which worked fine and implemented easy. Fingerprinting method has been a popular research direction so far. 
The research of Huang ChengLiang, Chen Wei ${ }^{[5,6]}$ and so on used the acceleration signal to measure pedestrians' walking step, which used the models to estimate step size and the course signal to calculate the information about the location, distance and direction of the pedestrians. The problem was decomposed into Step Detection, Step Length Estimation and Heading Determination. Precision of inertial sensors was not so important to PDR.

\section{Methods}

\section{Fingerprinting positioning technology based on WLAN.}

Fingerprinting positioning technology based on WLAN has been a leading indoor positioning method at present. There are two main phases in Fingerprinting positioning process, they are offline phase and online phase.

(1) Offline phase

The target of Offline phase is to establish a positioning fingerprinting database. Its job is mainly gathering fingerprint feature information-RSS of RP's (reference point) position in region of interest. Mobile terminal measures RSS from different AP in each reference point one by one. Meanwhile mobile terminal will store the corresponding MAC address and coordinate information in database, until spreading all over whole region of interest.

(2) Online phase

Mobile terminal measures RSS of AP around, and matching calculates them with RSS vector stored in database beforehand. Matching principle is according to Euclidean distance of RSS, as formula (1) shows:

$$
D_{j}=\sqrt{\sum_{i=1}^{n}\left(r S S_{i}-R S S_{j i}\right)^{2}}
$$

In the formula, $D_{j}$ is Euclidean distance of RSS between reference point $\mathrm{j}$ and mobile terminal. The smaller $D_{j} \mathrm{~A}$ is, the shorter the distance between them is. And $r s s=\left(r S S_{1}, r S S_{2}, \cdots, r S S_{n}\right)$ vector represents RSS of $\mathrm{n}$ APs measured by mobile terminal, $\mathrm{RSS}=\left(R S S_{j 1}, R S S_{j 2}, \cdots, R S S_{j n}\right)$ vector represents fingerprinting information of reference point $\mathrm{j}$.

Choosed $\mathrm{m}$ the smallest reference points of Euclidean distance $\left(\mathrm{x}_{1}, \mathrm{x}_{2}, \cdots, \mathrm{x}_{\mathrm{m}}\right)$, linear weighting of reference point coordinates are used to represent current position coordinates of mobile subscribers $\mathrm{x}_{0}=\left(x_{0}, y_{0}\right)$, as calculated formula shows:

$$
\mathbf{x}_{0}=\sum_{k=1}^{m} \omega_{k} \mathbf{x}_{\mathrm{k}} \quad \mathbf{x}_{0}=\sum_{k=1}^{m} \omega_{k} \mathbf{x}_{\mathrm{k}}
$$

In the formula, $\omega_{k}$ is weight of reference point $\mathrm{k}$, as calculated formula shows:

$$
\omega_{k}=\frac{\frac{1}{D_{k}}}{\sum_{k=1}^{m} \frac{1}{D_{k}^{2}}}
$$

We can know through the analysis above that Fingerprinting positioning method is based on assuming that RSS of fixed position is a stable value to achieve high precision positioning. In fact, due to the influence of multipath interference factors, that RSS of fixed position can float up to $20 \mathrm{db}$ deteriorates accuracy of WLAN positioning seriously. Meanwhile, in the area without AP, when WLAN positioning is failed, the supplementation of other positioning systems will be needed.

PDR based on Inertial sensors.

PDR algorithm concluded Step Detection, Step Length Estimation and Heading Determination ${ }^{[7]}$. It used step length and position to calculate the relative position of pedestrians from the known initial position. 
(1) Step Detection

In every step, pedestrians' feet would experience a periodic change process of steadying, accelerating, decelerating and steadying again ${ }^{[8]}$. In this paper, acceleration data was used to carry out step detection by the combination of zero point detection, peak detection and time limit.

When pedestrian walked, three axis of acceleration signal all appeared periodic features. Total acceleration value of three axis was used to detect steps. The amplitude of total acceleration value in the $\mathrm{i}^{\text {th }}$ sampling was:

$$
a_{i}=\sqrt{\left(a_{i}^{x}\right)^{2}+\left(a_{i}^{y}\right)^{2}+\left(a_{i}^{z}\right)^{2}}
$$

When pedestrian walked, distortion of acceleration signal caused by the physical shock and other reasons would affect the detection step, so we used the ripple filter to reduce the influences of external interference ${ }^{[9]}$.

$$
a_{i}=\frac{\sum_{i=i-N}^{i+N} a_{i}}{2 N+1}
$$

The " $2 \mathrm{~N}+1$ " was the length of filter window. Finding out the local peak value though comparing three adjacent acceleration values, if the local peak value was greater than the certain threshold, it would be the effective peak. If the interval was greater than $300 \mathrm{~ms}$ though calculating the time interval between two adjacent effective peaks, it indicated we had detected steps.

(2) Step Length Estimation

In biomechanics, step length increases with the increase of step frequency. When the frequency is $1.35 \sim 2.45$, step length can be shown as ${ }^{[10,11]}$ :

$$
S=0.4504 f-0.1656
$$

In this formula, $\mathrm{f}$ is frequency and $\mathrm{s}$ is step length.

(3) Heading Determination

We could get the direction angle relative to the magnetic north pole from magnetometer. As the picture showed, four directions could be distinguished by magnetometer.

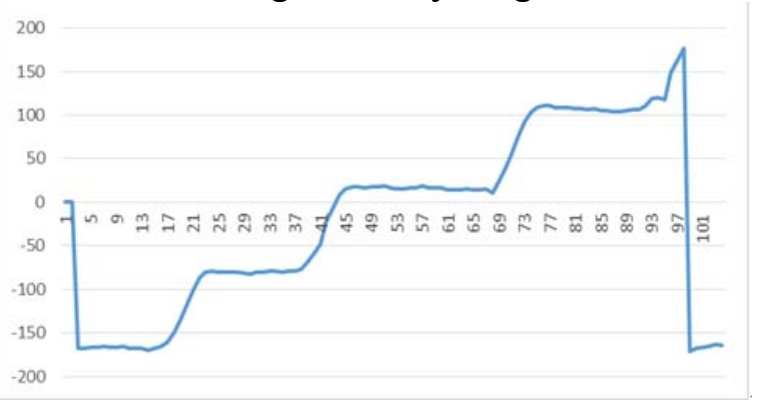

Pic 1 Magnetometer information

WLAN/inertial sensors combined positioning system.

A data fusion algorithm based on particle filter was designed in this paper. Complementary features of two systems was used to bring about an enhanced WLAN/inertial sensors combined positioning system, as picture 2 shown.

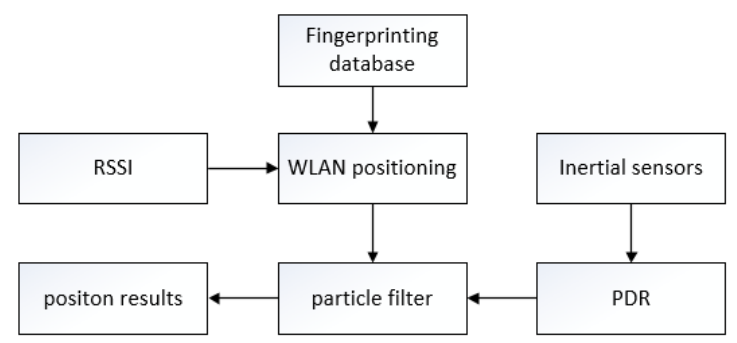

Pic 2 WLAN/inertial sensors combined positioning system based on particle filter

Particle filter ${ }^{[12,13]}$ was a sequential Monte Carlo method based on recurrence calculus. A particle set with correlation weights selected randomly from the probability density function was used to approach the posterior probability density function: 


$$
\operatorname{Pr}\left(\mathbf{x}_{k} \mid \mathbf{Z}_{0: k}\right)=\sum_{i=1}^{N} \omega_{k}^{i} \delta\left(\mathbf{x}_{k}-\mathbf{x}_{k}^{i}\right)
$$

In this formula, $\mathrm{x}_{k}$ was the state vector of target at time $\mathrm{k}, \mathrm{Z}_{0: k}$ was the measured value series before time $\mathrm{k}+1, \quad \mathbf{x}_{k}^{i}$ was the ith particle, $\omega_{k}^{i}$ was its weight, and $\mathrm{N}$ was amount of particle. The particle filter used here was decomposed into four steps.

\section{Step 1 Initialization}

$\mathrm{N}$ particles $\left\{\mathrm{x}_{0}^{\mathrm{i}}, i=1, \cdots, N\right\}$ could be produced by the initial probability density function $\operatorname{Pr}\left(\mathrm{x}_{0}\right) . \operatorname{Pr}\left(\mathrm{x}_{0}\right)$ used Gauss distribution and its mean value was the target initial position.

\section{Step 2 Forecast}

Particle filter forecasted the next step in the target location information $\left(x_{k+1}, y_{k+1}\right)$ though combining the data processing results of inertial sensors. The formula was shown as:

$$
\left[\begin{array}{c}
x_{k+1} \\
y_{k+1}
\end{array}\right]=\left[\begin{array}{lll}
1 & 0 & \sin \left(\phi_{k}\right) \\
0 & 1 & \cos \left(\phi_{k}\right)
\end{array}\right]\left[\begin{array}{c}
x_{k} \\
y_{k} \\
S
\end{array}\right]
$$

In this formula, $\left[\begin{array}{ll}x_{k+1} & y_{k+1}\end{array}\right]^{T}$ was the state vector of every particle, $\phi_{k}$ was the direction angle of pedestrians relative to magnetic north pole got by the data of inertial sensor. The "s" was the distance got by using data of inertial sensor to carry out step detection and step size estimation in the time interval between the WLAN positions in the $\mathrm{k}^{\text {th }}$ time and the $(\mathrm{k}+1)^{\text {th }}$ time.

Step 3 Weight calculation and normalization

Weight of particles was calculated by measurement model and Current observation value:

$$
\begin{gathered}
\omega_{k+1}^{i}=\operatorname{Pr}\left[\mathrm{Z}_{k+1} \mid \mathbf{x}_{k}^{i}\right]=\frac{1}{\sqrt{2 \pi} \sigma} \exp \left[-\frac{|| \mathbf{x}_{k}^{z}-\mathbf{x}_{k}^{i}||}{2 \sigma^{2}}\right] \\
\omega_{k+1}^{i}=\frac{\omega_{k+1}^{i}}{\sum_{j=1}^{N} \omega_{k+1}{ }^{i}}
\end{gathered}
$$

In this formula, $Z_{k+1}$ was current surveyed RSS by target. The current estimation of the Location of target, shown as $\mathbf{x}_{k}^{z}$, could be got by Fingerprinting positioning method presented formerly. $\mathrm{x}_{k}^{i}$ was the positioning information of the $\mathrm{i}^{\text {th }}$ particle in time $\mathrm{k}$. Noise variance shown as $\sigma$ was chosen though actual floating variance of RSS. Formula(10) showed that when the particle's position was closer to the target's current estimate position, the weight of the particle was greater to obtain an accurate posterior probability distribution.

\section{Step 4 Resampling}

Resampling ${ }^{[14]}$ was the key of particle filter. According to the probability density function $\operatorname{Pr}\left(\mathbf{x}_{k} \mid \mathbf{z}_{k}\right)$ which produced N new particle $\left\{\mathbf{x}_{k}^{\mathrm{i}}, i=1, \cdots, N\right\}$, resampling could be used to solve the problem of particle degradation

\section{Experimental Results}

We chose the New Main Building of BeiHang University for the experiment site. In the structure chart as pic 3 shown, there were 13 Aps in positioning area. The black points at an interval of 4 meters, which were on behalf of RP, were used to set up a database of WLAN positioning system fingerprint information database. The black dotted lines were on behalf of real experimental paths. 


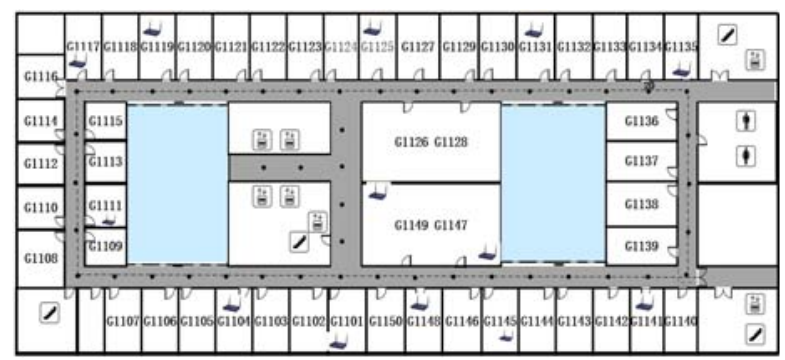

Pic 3 Experiment site and experimental paths

The mobile terminal that moved along by experimental paths at a speed of $1 \mathrm{~m} / \mathrm{s}$ collected RSS of AP and inertial sensors data to conduct experiments of WLAN position, inertial navigation and WLAN/ inertial sensors combined position. From the comparison between WLAN position results and real paths in pic 4, we found that the path got by WLAN position often happened jumps. It was as a result of RSS floating caused by multipath interference and affected the performance of WLAN system badly.

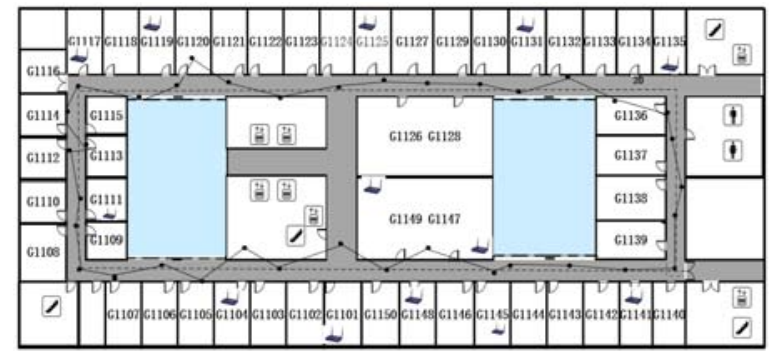

Pic 4 Real paths and WLAN position results

From the comparison between inertial navigation results and real paths in pic 5 , we found that the path got by inertial navigation was stable without jumps and had a good positioning accuracy in a short time. But as positioning time went on, due to affect of the accumulative error, accuracy of inertial navigation was getting worse and the positioning path deviated the real path seriously.

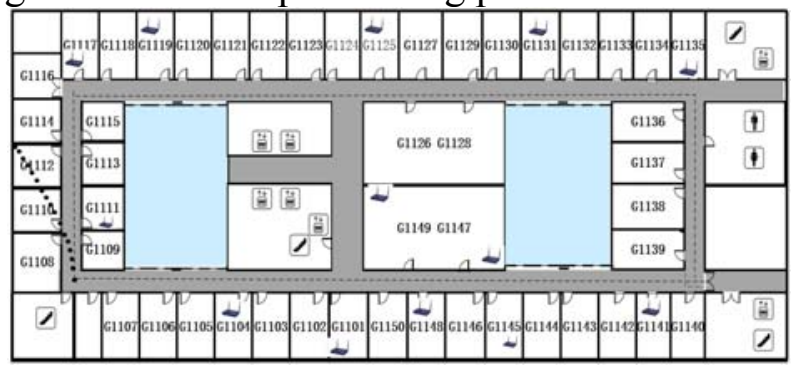

Pic 5 Real paths and inertial navigation results

The comparison between WLAN/inertial sensors combined position results and real paths was presented in pic 6 . The combined positioning system based on data fusion observably improved all sorts of problems in WLAN position and inertial navigation. Improvements could be shown as that jump and accumulative error was restrained primely, positioning path was stable, and accuracy of positioning was raised.

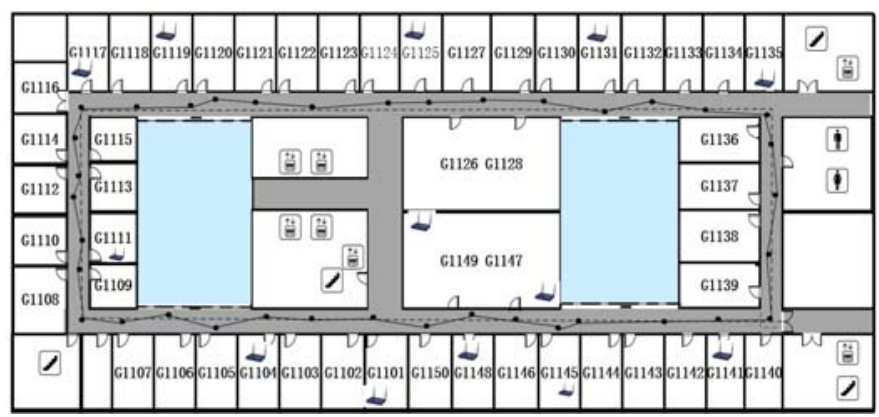

Pic 6 Real paths and WLAN/inertial sensors combined position results

Meanwhile, the comparison of performance between the positioning systems presented in this thesis and other indoor positioning systems (such as Triangulation, RADAR (radio detection and ranging) simulation and so on) was listed in sheet 1 . 
Sheet 1 Performance compared with other positioning system

\begin{tabular}{|c|c|c|c|c|}
\hline System & $\begin{array}{c}\text { proposed } \\
\text { system }\end{array}$ & Triangulation & RADAR & simulation \\
\hline $\mathrm{RMSE} / \mathrm{m}$ & 1.8 & 5.2 & 3.5 & 4.7 \\
\hline
\end{tabular}

The accumulated probability distributions of positioning error of WLAN/inertial sensors combined position, WLAN position and inertial navigation was presented in pic 7 . The root mean square error (RMSE) of inertial navigation was $15.8 \mathrm{~m}$; WLAN position's was $3.9 \mathrm{~m}$; The RMSE of WLAN/inertial sensors combined position presented in the thesis was $1.8 \mathrm{~m}$.

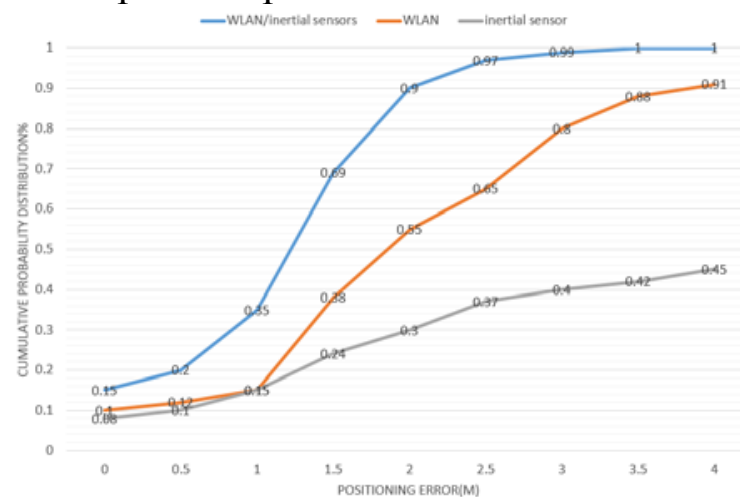

Pic 7 Positioning error cumulative probability distribution of different methods

\section{Conclusion}

The data fusion architecture based on particle filter brought out in this paper, achieved WLAN/inertial sensors combined positioning system based on intelligent terminal whose outstanding advantages were the significant improvement of the positioning accuracy of the system and the effective extension of the application range of the system. As the result of experiment shown, the proposed system was reduced by $49 \%$ and $88 \%$ as compared with WLAN based system and inertial sensors based system. Meanwhile compared with other indoor positioning systems, the system had higher positioning accuracy and reliability.

\section{Acknowledgement}

This research was supported by National 863 Grant No. 2015AA124103.

\section{References}

[1] Fang S H, Lin T N, Lee K C. A Novel Algorithm for Multipath Fingerprinting in Indoor WLAN Environments[J]. Wireless Communications IEEE Transactions on, 2008, 7(9):3579-3588.

[2] Youssef M, Agrawala A. The Horus WLAN location determination system[C]//Proceedings of the 3rd international conference on Mobile systems, applications, and services. ACM, 2005: 205-218.

[3] Huang C, Liao Z, Zhao L. Synergism of INS and PDR in Self-Contained Pedestrian Tracking With a Miniature Sensor Module[J]. Sensors Journal IEEE, 2010, 10(8):1349-1359.

[4] Bahl P, Padmanabhan V N. RADAR: An in-building RF-based user location and tracking system[C]//INFOCOM 2000. Nineteenth Annual Joint Conference of the IEEE Computer and Communications Societies. Proceedings. IEEE. Ieee, 2000, 2: 775-784.

[5] Chen W, Fu Z, Chen R, et al. An integrated GPS and multi-sensor pedestrian positioning system for 3D urban navigation[C]// Urban Remote Sensing Event, 2009 JointIEEE, 2009:1-6.

[6] Chen W, Chen R, Chen Y, et al. An adaptive calibration approach for a 2-axis digital compass in 
a low-cost pedestrian navigation system[C]// Instrumentation and Measurement Technology Conference (I2MTC), 2010 IEEEIEEE, 2010:1392-1397.

[7] Sun Z, Mao X, Tian W, et al. Activity classification and dead reckoning for pedestrian navigation with wearable sensors[J]. Measurement Science \& Technology, 2009, 20(1):187-188.

[8] Ding Q C, Xiong A B, Zhao X G, et al. A novel EMG-driven state space model for the estimation of continuous joint movements[C]// Systems, Man, and Cybernetics (SMC), 2011 IEEE International Conference onIEEE, 2011:2891-2897.

[9] Liu Z, Aduba C, Won C H. In-plane dead reckoning with knee and waist attached gyroscopes[J]. Measurement, 2011, 44(10):1860-1868.

[10] Rahman M S, Park Y, Kim K D. RSS-based indoor localization algorithm for wireless sensor network using generalized regression neural network[J]. Arabian journal for science and engineering, 2012, 37(4): 1043-1053.

[11] Liu H, Darabi H, Banerjee P, et al. Survey of wireless indoor positioning techniques and systems[J]. Systems, Man, and Cybernetics, Part C: Applications and Reviews, IEEE Transactions on, 2007, 37(6): 1067-1080.

[12] Chan E C L, Baciu G, Mak S C. Using Wi-Fi Signal Strength to Localize in Wireless Sensor Networks $[\mathrm{C}] / /$ Communications and Mobile Computing, International Conference onIEEE, 2009:538-542.

[13] Francois, Spies, Soumaya, et al. WiFi GPS based combined positioning algorithm[C]// Wireless Communications, Networking and Information Security (WCNIS), 2010 IEEE International Conference onIEEE, 2010:684-688.

[14] ZHOU Mu, XU Yu Bin, MA Lin, TIAN Shuo. On the statistical errors of RADAR location sensor networks with built-in Wi-Fi Gaussian linear fingerprints[J]. Sensors, 2012, 12(3): 3605-3626. 